\title{
Gestational weight gain in Chinese women - results from a retrospective cohort in Changsha, China
}

\author{
Xin Huang ${ }^{1}$, Hongzhuan Tan ${ }^{2}$, Ming $\mathrm{Cai}^{3}$, Ting Shi ${ }^{4}$, Chunmei $\mathrm{Mi}^{5^{*+}}$ and Jun Lei ${ }^{5^{* \dagger}}$ (D)
}

\begin{abstract}
Background: The generalizability of the gestational weight gain (GWG) ranges recommended by the Institute of Medicine (IOM) to Chinese women is disputed.

Methods: In 2016, 16,780 pregnant women who gave birth to live singletons in Changsha, China, were enrolled. First, subjects with optimal pregnancy outcomes were identified for the GWG percentile distribution description and for comparison to the IOM recommendations. Second, all subjects with optimal GWG according to the IOM body mass index (BMI) cutoffs and those with optimal GWG according to the Asian BMI cutoffs were selected. Pregnancy outcomes were compared between those two groups.

Results: A total of 13,717 births with optimal pregnancy outcomes were selected to describe the GWG distribution. The height and central position of the GWG distributions determined by the Asian BMI cutoffs differed from those determined by the IOM BMI cutoffs among the overweight and obese groups. The recommended IOM GWG ranges were narrower than and shifted to the left of the observed distributions. In both BMI classification schemes, however, the IOM-recommended ranges were within the middle 70\% (Pc 15th-85th) and 50\% (Pc 25th-75th) of the observed distribution. A total of 6438 (38.37\%) and 6110 (36.41\%) women gained optimal GWG, according to the IOM and Asian BMI classifications, respectively. Compared with those with optimal GWG according to IOM BMI cutoffs, women with optimal GWG according to the Asian BMI cutoffs had lower risks of both macrosomia (adjusted $\mathrm{OR}=0.79,95 \% \mathrm{Cl}: 0.67-0.94$ ) and large-for-gestational age (adjusted $\mathrm{OR}=0.86,95 \% \mathrm{Cl}$ : 0.76, 0.98). However, no significantly different risks of preterm, low birthweight, small-for-gestational age, pregnancy-induced hypertension, or gestational diabetes were found between them.
\end{abstract}

Conclusions: The IOM-recommended GWG ranges are within the middle $70 \%$ of the distributions in Chinese women, and pre-pregnancy weight status should be determined by the Asian BMI cut-off points for monitoring and making GWG recommendations to Chinese women.

Keywords: Body mass index, Gestational weight gain, Chinese women, Pregnancy

\footnotetext{
*Correspondence: 615871972@qq.com; junlei.xy3yy@hotmail.com

${ }^{+}$Chunmei Mi and Jun Lei contributed equally to this work.

${ }^{5}$ Department of Obstetrics and Gynecology, The Third Xiangya Hospital of

Central South University, 138 Tongzipo Road, Yuelu District, Changsha

410013, Hunan, Province, China

Full list of author information is available at the end of the article
}

(c) The Author(s). 2018 Open Access This article is distributed under the terms of the Creative Commons Attribution 4.0 International License (http://creativecommons.org/licenses/by/4.0/), which permits unrestricted use, distribution, and reproduction in any medium, provided you give appropriate credit to the original author(s) and the source, provide a link to the Creative Commons license, and indicate if changes were made. The Creative Commons Public Domain Dedication waiver (http://creativecommons.org/publicdomain/zero/1.0/) applies to the data made available in this article, unless otherwise stated. 


\section{Background}

A nutritious diet during pregnancy maintains maternal energy requirements, provides a substrate for the development of new fetal tissues, and builds energy reserves for postpartum lactation [1]. Therefore, the importance of nutrition in pregnancy cannot be overemphasized. Previous studies have proved that excessive or inadequate weight gain during pregnancy has negative implications on pregnancy outcomes, putting the health of both mother and infant at risk [2-4].

To avoid maternal and infant adverse outcomes, in 2009, the Institute of Medicine (IOM), USA, published revised recommended gestational weight gain (GWG) [5] and the revisions was based on pre-pregnancy weight status. Since the effect of weight gain during pregnancy on fetal growth has found to be varied by maternal pre-pregnancy body mass index (BMI) $[6,7]$ In Jan 2018, the national prenatal care guideline for Chinese women was officially published, but it just duplicated the IOM recommendations for GWG monitoring among Chinese [8]. Those recommendations, however, were made based on Western populations, and their generalizability to Chinese women is under dispute [9-13].

One of the reasons for the conflict may be that BMI cut-off points used in previous studies are inconsistent: Yang et al. [9] used IOM-recommended BMI categories and concluded that IOM GWG recommendations are suitable for Chinese; Zhou et al. [10] and Wong et al. [11] used the Asian BMI cut-off points and found that the IOM GWG recommendations are inappropriate. The weight gain recommendations of the IOM are based on Western BMI cut-off points [5]. However, the Asian BMI cut-off points for determining overweight and obesity in Asian populations are different [13] because researchers found that Asian populations have different associations between BMI and health risks than do Western populations [14, 15]. Under different BMI cut-off points related to different recommended weight gain ranges [5], some Chinese women would be classified in lower pre-pregnancy BMI categories and assigned to larger target weight gain ranges when the IOM BMI cut-off points were used instead of the Asian BMI cut-off points. The appropriateness of using the IOM GWG recommendations for Chinese women should be examined by describing the distributions of GWG by pre-pregnant weight status, as determined using both Asia and IOM BMI cut-off points.

We therefore conducted a retrospective pregnancy cohort study in Changsha, China, 1) to describe the GWG distributions by BMI group, as determined using the IOM and Asian BMI cut-off points, and compare those distributions to the IOM GWG recommended ranges among women with optimal birth outcomes and 2) to compare the pregnancy outcomes among those with the optimal GWG determined using the Asian BMI cut-off points to those with the optimal GWG determined using the IOM BMI cut-off points.

\section{Methods \\ Study population}

Our population-based retrospective cohort study was conducted in Changsha city in Hunan Province. Changsha is a city with 6 urban districts (Yuelu, Tianxin, Kaifu, Yuhua, Furong, and Wangcheng district), 1 county (Changsha county) and 2 county-level cities (Ningxiang and Liuyang city). In 2016, a total of 116,336 pregnant women gave birth in Changsha. All pregnant women aged 18 years or older $(18,843)$ who resided in Yuelu or Tianxin district and had a live birth from Jan to Dec 2016 were enrolled when they came to local maternity care units to apply for birth certificates. The sponsors of the study had no role in study design, data collection, data analysis, data interpretation, or writing the report. The study protocol was reviewed and approved by the Ethical and Confidentiality Committee of Central-South University and by both institutional review boards (IRB) from the Maternity and Child Care Hospital of Yuelu District and Tianxin District. All participants provided signed written consent. Women who had multiple births and/or had infants with birth defects, or who had chronic hypertension, diabetes, renal disease or cardiovascular diseases before pregnancy, or were lacking data on GWG were excluded, which yielded a final eligible analytical sample size of 16,780 .

\section{Data collection and variable definition}

All data used in the present study were extracted from two sources: antenatal care booklets and hospital discharge abstracts (including both maternal obstetrical delivery records and newborn hospital records). In China, medical information on antenatal care is routinely recorded by certified doctors or nurses in an antenatal care booklet beginning with the first prenatal visit. The booklet is kept by the individual during pregnancy and must be returned to the local maternity care unit, along with the hospital discharge abstract, before applying for an official birth certificate at the hospital. General information on maternal demographic characteristics, pre-pregnancy weight and obstetric history were reported by the interviewee themselves, which could be obtained from antenatal care booklets. Information on weight at delivery, maternal complications and neonatal outcomes was extracted from discharge abstracts.

Pre-pregnancy BMI was calculated as maternal pre-pregnancy weight in kilograms divided by squared height in meters. GWG was calculated by subtracting pre-pregnancy weight from maternal weight at delivery in kilograms. The optimal GWG was defined as the weight gain during pregnancy within the IOM recommended range by pre-pregnancy BMI category. Pre-pregnancy weight 
status was categorized using both the Asian and IOM cut-off points (Table 1).

Adverse pregnancy outcomes considered in this study included preterm (delivered at less than 37 weeks of gestation), low birthweight (LBW, birthweight $<2500 \mathrm{~g}$ ), macrosomia (birthweight $\geq 4000 \mathrm{~g}$ ), large-for-gestational age (LGA, birthweight $>90$ th for gestational age and sex), small-for-gestational age (SGA, birthweight $<10$ th percentile for gestational age and sex), pregnancy-induced hypertension (PIH) and gestational diabetes mellitus (GDM). For infants born between 28 and 44 weeks of gestation, birthweight reference percentiles for Chinese infants [16] were used to define SGA and LGA. For infants born between 22 and 27 weeks of gestation, a United States national reference was applied [17]. PIH included gestational hypertension and preeclampsia which was defined as systolic BP (SBP) $\geq 140 \mathrm{mmHg}$ and/or diastolic BP (DBP) $\geq 90 \mathrm{mmHg}$, occurring for the first time after 20 weeks of gestation, with or without proteinuria. Oral glucose tolerance test (OGTT) was routinely examined at 24-28 gestational weeks, and GDM was defined as diabetes that was first diagnosed during pregnancy, with 3-h 100 g OGTT results exceeding cut-offs for two or more values: fasting plasma glucose $\geq 5.3 \mathrm{mmol} / \mathrm{L}, 1-\mathrm{h} \geq 10.0 \mathrm{mmol} / \mathrm{L}, 2-\mathrm{h} \geq 8.6 \mathrm{mmol} / \mathrm{L}$ and 3-h $\geq 7.8 \mathrm{mmol} / \mathrm{L}$ ).

\section{Statistical analysis}

Our analysis has two parts (the analytical scheme is shown in Fig. 1). First, to describe the GWG distribution among Chinese women, subjects $(n=13,717)$ with an optimal pregnancy outcome were identified. A subject with an optimal outcome in our study was defined as a pregnant woman without prenatal medical complications (such as GDM or PIH), giving live birth at term (gestational age between 37 and 42 weeks), and with infant birth weight between 2500 and 3999 g. Among those with an optimal outcome, analysis of variance (ANOVA) test was used to examine the central tendency and variability for GWG by different maternal characteristics, including maternal age, parity, year of education, smoking during pregnancy and pre-pregnancy BMI group. The Student- Newman Keuls (SNK) test was adopted to make multiple comparisons when group categories were greater than two. The percentile distributions (5th, 15th,

Table 1 IOM weight gain recommendations for pregnancy by pre-pregnancy weight status

\begin{tabular}{llll}
\hline $\begin{array}{l}\text { Weight } \\
\text { category }\end{array}$ & $\begin{array}{l}\text { IOM BMl } \\
\text { category criteria }\end{array}$ & $\begin{array}{l}\text { Asian BMI } \\
\text { category criteria }\end{array}$ & $\begin{array}{l}\text { IOM-recommended } \\
\text { weight gain }\end{array}$ \\
\hline Underweight & $<18.5 \mathrm{~kg} / \mathrm{m}^{2}$ & $<18.5 \mathrm{~kg} / \mathrm{m}^{2}$ & $12.5-18 \mathrm{~kg}$ \\
Normal weight & $18.5-24.9 \mathrm{~kg} / \mathrm{m}^{2}$ & $18.5-22.9 \mathrm{~kg} / \mathrm{m}^{2}$ & $11.5-16 \mathrm{~kg}$ \\
Overweight & $25.0-29.9 \mathrm{~kg} / \mathrm{m}^{2}$ & $23-24.9 \mathrm{~kg} / \mathrm{m}^{2}$ & $7-11.5 \mathrm{~kg}$ \\
Obese & $\geq 30 \mathrm{~kg} / \mathrm{m}^{2}$ & $\geq 25 \mathrm{~kg} / \mathrm{m}^{2}$ & $5-9 \mathrm{~kg}$ \\
\hline
\end{tabular}

25th, 50th, 75th, 85th, and 95th) of GWG according to both the Asian and IOM pre-pregnancy BMI cut-off points were compared. Observations regarding percentile trends were made on a descriptive basis, and statistical tests for trend were not reported. The middle 70\% (Pc 15th-85th) and 50\% (Pc 25th-75th) of the observed GWG distribution was compared to the IOM ranges.

Second, to examine which BMI classification scheme is more suitable for Chinese women, all subjects with an optimal GWG determined by the IOM BMI cut-off points $(n=6438)$ and those with optimal GWG determined by the Asian BMI cut-off points $(n=6110)$ were selected. Pregnancy outcomes were compared between those two groups. The crude odds ratios (OR) and 95\% confidence intervals $(\mathrm{CI})$ were calculated. Multivariate logistic regression models were also adopted to calculate adjusted OR and 95\%CI. Potential confounding variables included maternal age $(<25,25-34$, and $\geq 35$ years, 25 34 as reference), parity (primiparous or multiparous), year of education ( $\leq 12$ years or $>12$ years) and smoking during pregnancy (yes or no). Subgroup analyses were conducted among subjects with a pre-pregnancy BMI in the 23-24.9 and 25-29.9 strata. Statistical significance was assessed at the 0.05 level (two-tailed test). All analyses were performed using the SAS software, version 9.2 (SAS Institute, Inc., Cary, NC).

\section{Results}

A total of 16,780 qualified subjects were enrolled in our study. Of them, 603 (3.59\%) mothers were diagnosed with $\mathrm{PIH}$, and 176 (1.05\%) were diagnosed with GDM; 1249 (7.44\%) births were preterm, 1001 (5.97\%) were low birthweight, 1030 (6.14\%) were macrosomia, 1389 (8.28\%) were SGA and 1984 (11.82\%) were LGA.

Based on IOM pre-pregnancy BMI cut-off points, there were 2651 (15.80\%), 12,272 (73.13\%), 1575 (9.39\%) and 282 (1.68\%) women defined as underweight, normal weight, overweight and obese, respectively, and 6438 (38.37\%) women had an optimal GWG. According to Asian BMI cut-off points, there were 2651 (15.80\%), 9897 (58.98\%), 2375 (14.15\%) and 1857 (11.07\%) women classified as underweight, normal weight, overweight and obese, respectively, and 6110 (36.41\%) women had an optimal GWG.

\section{Weight gain during pregnancy}

After excluding those with preterm, LBW, macrosomia, PIH or GDM, 13,717 births with an optimal pregnancy outcome were selected to describe the distribution of GWG. Of them, the mean maternal age was $27.3 \pm 4.44$ years, the mean infant birthweight was $3274.9 \pm 780.24 \mathrm{~g}$, the mean pre-pregnancy BMI was $21.3 \pm 3.15 \mathrm{~kg} / \mathrm{m}^{2}$, and the mean GWG was $14.4 \pm 5.39 \mathrm{~kg}$.

GWG was found to vary significantly by maternal age, parity, year of education, smoking during pregnancy, 


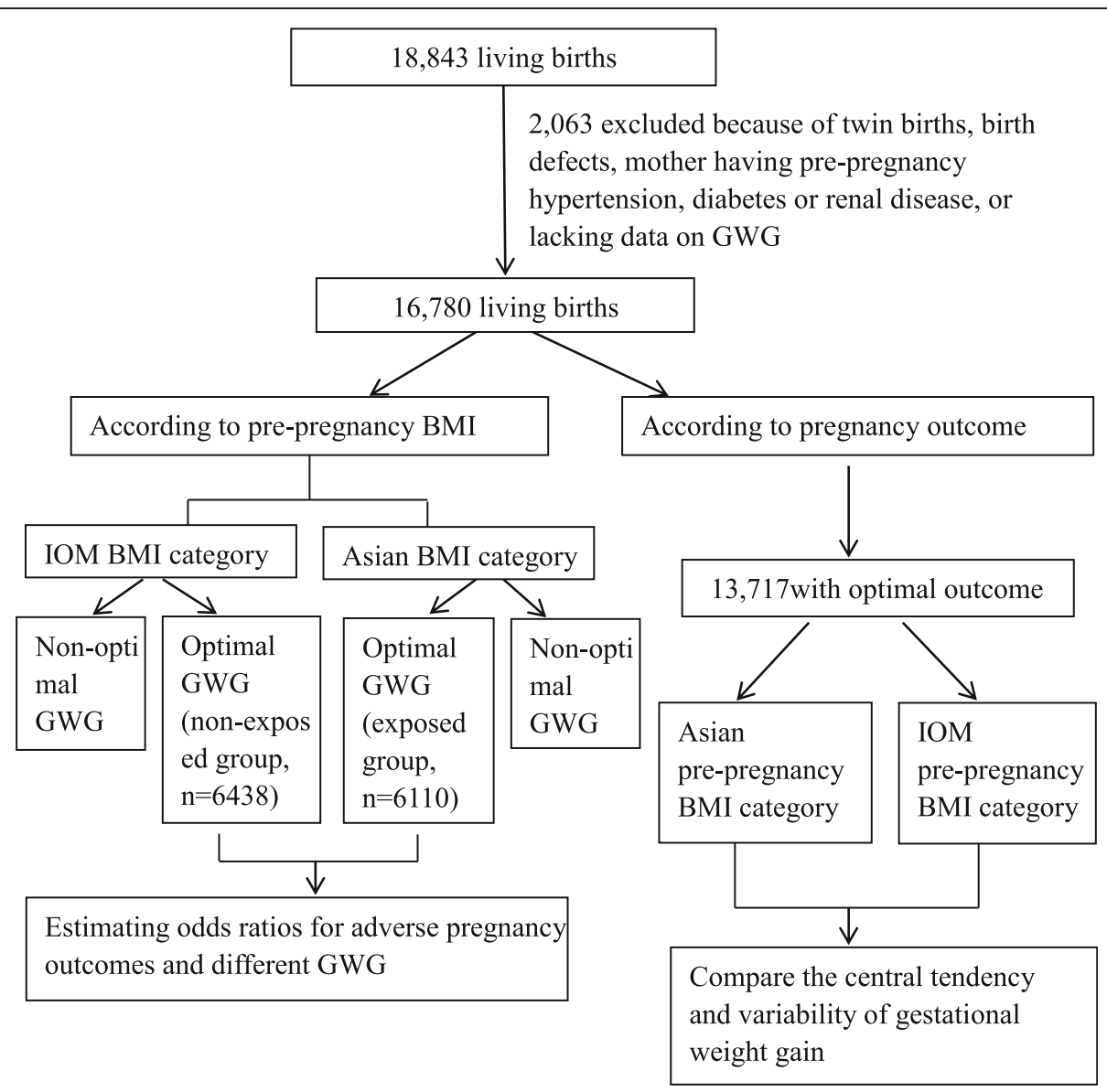

Fig. 1 Analytical scheme of present study

and both IOM and Asian pre-pregnancy BMI category (Table 2). Mothers who were 25-34 years old, primiparous, educated for less than 12 years, or smoked during pregnancy had a greater GWG than did the others. The relationship between GWG and pre-pregnancy BMI varied when different BMI classification schemes were used. Based on the Asian BMI classification scheme, the mean GWG decreased as pre-pregnancy BMI increased; the underweight group had the highest mean GWG, whereas the obese group had the lowest. However, when the IOM classification scheme was adopted, the relationship between GWG and pre-pregnancy BMI followed a U-shaped curve, and the overweight group had the lowest mean GWG (Table 2). The GWG percentile distribution analysis showed the same results (Table 3). Compared with the IOM BMI classification schemes, the shape and width of the GWG distributions resulting from the Asian BMI classification scheme were nearly identical for the underweight and normal weight groups, whereas curves for the overweight and obese groups differed in both height and the central position of the distribution (Table 3, Fig. 2).

As shown in Table 3 and Fig. 2, the IOM recommendations by pre-pregnancy weight status are very narrow compared to the GWG distributions observed for Chinese women. In the both BMI classification schemes, the IOM recommended ranges are within the middle 70 and $50 \%$ of the observed distributions. Among underweight and normal weight women, the recommended IOM GWG ranges fall near the middle of the distribution. However, among overweight and obese women, the IOM recommended GWG ranges fall within the lower half of the distribution (Fig. 2).

\section{Adverse pregnancy outcomes and optimal GWG by different BMI classification}

Compared with those with optimal GWG determined by IOM BMI cut-off points, women with optimal GWG determined by the Asian BMI cut-off points had lower risks of having macrosomia (crude OR $=0.79,95 \% \mathrm{CI}$ : $0.67-0.94$ ) and LGA infant (crude $\mathrm{OR}=0.86,95 \% \mathrm{CI}$ : $0.76,0.97$ ) (Table 4). After adjustments for maternal age, education, parity and smoking status during pregnancy, the associations still significantly exist. No significantly different risks of having preterm, LBW or SGA infants or different risks of having PIH or GDM were found between women with an optimal GWG determined by 
Table 2 Gestational weight gain by maternal characteristics among subjects with optimal pregnancy outcome ${ }^{c}$ in Changsha, Hunan, China, in 2016

\begin{tabular}{|c|c|c|c|c|c|c|}
\hline \multirow[t]{2}{*}{ Characteristics } & \multirow[t]{2}{*}{ N (\%) } & \multicolumn{4}{|c|}{ Gestational weight gain } & \multirow[t]{2}{*}{$p$} \\
\hline & & Mean, kg & SD & Min/Max & $C V, \%$ & \\
\hline Overall subjects & $13,717(100 \%)$ & 14.3 & 5.31 & $-4.5 / 48.5$ & 37.00 & \\
\hline \multicolumn{7}{|l|}{ Maternal age (years) } \\
\hline$\leq 24$ & $3669(25.75)$ & $13.3^{d}$ & 5.74 & $-4.5 / 31.8$ & 43.27 & \multirow[t]{3}{*}{$<0.001$} \\
\hline $25-34$ & $8724(63.60)$ & $14.8^{\mathrm{d}}$ & 5.11 & $-4.5 / 48.5$ & 34.58 & \\
\hline$\geq 35$ & $1324(9.65)$ & $14.5^{\mathrm{d}}$ & 4.90 & $-3.0 / 34.0$ & 33.87 & \\
\hline \multicolumn{7}{|l|}{ Parity } \\
\hline Primiparous & $9549(69.61)$ & 14.9 & 4.93 & $-4.5 / 48.5$ & 32.97 & \multirow[t]{2}{*}{$<0.001$} \\
\hline Multiparous & $4168(30.39)$ & 12.9 & 5.85 & $-4.5 / 31.5$ & 45.17 & \\
\hline \multicolumn{7}{|l|}{ Education (years) } \\
\hline$\leq 12$ & $8701(64.37)$ & 15.3 & 4.60 & $-4.5 / 32.5$ & 21.19 & \multirow[t]{2}{*}{$<0.001$} \\
\hline$\geq 13$ & $5016(36.57)$ & 12.6 & 5.97 & $-4.5 / 48.5$ & 47.29 & \\
\hline \multicolumn{7}{|c|}{ Smoking during pregnancy } \\
\hline Yes & $1461(10.65)$ & 15.7 & 4.27 & $-3.5 / 29.0$ & 27.21 & \multirow[t]{2}{*}{$<0.001$} \\
\hline No & $12,256(89.35)$ & 14.2 & 5.40 & $-4.5 / 48.5$ & 38.05 & \\
\hline \multicolumn{7}{|c|}{ IOM pre-pregnancy BMI category ${ }^{a}$} \\
\hline Underweight & $2261(16.48)$ & $15.8^{d}$ & 4.36 & $-4.5 / 31.5$ & 27.54 & \multirow[t]{4}{*}{$<0.001$} \\
\hline Normal weight & $10,114(73.73)$ & $14.3^{d}$ & 5.19 & $-4.5 / 40.0$ & 36.20 & \\
\hline Overweight & $1149(8.38)$ & $11.8^{\mathrm{d}}$ & 6.54 & $-4.5 / 48.5$ & 55.52 & \\
\hline Obese & $193(1.41)$ & $13.1^{d}$ & 7.01 & $-4.0 / 29.5$ & 53.66 & \\
\hline \multicolumn{7}{|c|}{ Asian pre-pregnancy BMI category ${ }^{b}$} \\
\hline Underweight & $2261(16.48)$ & $15.8^{\mathrm{d}}$ & 4.36 & $-4.5 / 31.5$ & 27.54 & \multirow[t]{4}{*}{$<0.001$} \\
\hline Normal weight & $8273(60.31)$ & $14.7^{d}$ & 5.04 & $-4.0 / 40.0$ & 34.29 & \\
\hline Overweight & $1841(13.42)$ & $12.7^{d}$ & 5.56 & $-4.5 / 32.5$ & 43.62 & \\
\hline Obese & $1342(9.78)$ & $12.0^{\mathrm{d}}$ & 6.62 & $-4.5 / 48.5$ & 55.35 & \\
\hline
\end{tabular}

a IOM BMI category: underweight (BMI < 18.5), normal weight (BMI 18.5-24.9), overweight (BMI 25-29.9), obese (BMI $\geq 30)$

${ }^{\mathrm{b}}$ Asian BMI category: underweight (BMI < 18.5), normal weight (BMI 18.5-22.9), overweight (BMI 23-24.9), obese (BMI $\left.\geq 25\right)$

'Optimal outcome was defined as woman who has no prenatal medical complications (such as GDM or PIH), giving live birth at a gestational age between 37 and 42 weeks, and infant birth weight between 2500 and $3999 \mathrm{~g}$

${ }^{\mathrm{d}}$ SNK test showed significant differences between each other, $p<0.05$

different BMI cut-off points (Table 4). The subgroup analysis in the pre-pregnancy BMI 23.0-24.9 and 25-29.9 strata showed the same results (Additional file 1: Table S1 and Additional file 2: Table S2).

\section{Discussion}

Our data found that the GWG distribution for Chinese women varied with the use of different BMI classification schemes. The recommended IOM weight gain ranges are narrower than and shifted to the left of the actual distributions of GWG for Chinese women. We support the use of the Asian BMI categories to recommend and monitor target weight gains for Chinese pregnancies since lower risks of macrosomia and LGA were observed among those with an optimal GWG determined by the Asian BMI cut-off points.

\section{Weight gain during pregnancy among Chinese women}

The GWG guidelines issued by the IOM are intended for use among American women but not for other women who are substantially shorter or thinner than American women [5, 18-20]. Studies validating these guidelines among Chinese women are emerging and have reached inconclusive results [9-13]. Yang et al. [9], using the IOM-recommended BMI cut-off points and combining the overweight and obese groups, concluded that IOM GWG recommendations are suitable for Chinese women. Jiang et al.[12] also used IOM BMI cut-off points but concluded, however, that the IOM-recommended GWG range is too high for the Chinese population pregnant with singletons. Zhou et al.[10] and Wong et al. [11], using Asian BMI cut-off points, found that the IOM GWG recommendations are narrower than observed among Chinese women. Yang S [13] enrolled 76,854 women in Wuhan and 
Table 3 Distribution of gestational weight gain by pre-pregnancy BMI among subjects with optimal pregnancy outcomes in Changsha, Hunan, China, in 2016

\begin{tabular}{|c|c|c|c|c|c|c|c|c|}
\hline \multirow[t]{2}{*}{ Subgroups } & \multicolumn{7}{|c|}{ Gestational weight gain $(\mathrm{kg})$ by percentile } & \multirow{2}{*}{$\begin{array}{l}\text { IOM } \\
\text { recommenc } \\
\text { ranges }(\mathrm{kg})\end{array}$} \\
\hline & 5th & 15th & 25th & 50th & 75th & 85th & 95th & \\
\hline Overall subjects $(n=12,564)$ & 5.0 & 9.0 & 11.0 & 15.0 & 18.0 & 19.0 & 22.5 & \\
\hline \multicolumn{9}{|c|}{ IOM pre-pregnancy BMI category ${ }^{a}$} \\
\hline Underweight $(n=2002)$ & 9.0 & 11.5 & 13.0 & 16.0 & 18.5 & 20.0 & 23.0 & $12.5-18.0$ \\
\hline Normal weight $(n=9308)$ & 5.0 & 9.0 & 11.0 & 15.0 & 17.5 & 19.0 & 22.5 & $11.5-16.0$ \\
\hline Overweight $(n=1084)$ & 0.0 & 4.5 & 7.5 & 12.5 & 16.0 & 18.0 & 21.5 & $7.0-11.5$ \\
\hline Obese $(n=170)$ & 2.5 & 5.0 & 8.0 & 13.0 & 17.5 & 20.0 & 26.0 & $5.0-9.0$ \\
\hline \multicolumn{9}{|c|}{ Asian pre-pregnancy BMI category ${ }^{b}$} \\
\hline Underweight $(n=2002)$ & 9.0 & 11.5 & 13.0 & 16.0 & 18.5 & 20.0 & 23.0 & $12.5-18.0$ \\
\hline Normal weight $(n=7564)$ & 6.0 & 10.0 & 11.6 & 15.0 & 18.0 & 19.0 & 22.8 & $11.5-16.0$ \\
\hline Overweight $(n=1744)$ & 3.0 & 7.0 & 9.5 & 13.0 & 16.5 & 18.0 & 21.0 & $7.0-11.5$ \\
\hline Obese $(n=1254)$ & 0.4 & 5.0 & 7.5 & 12.5 & 16.0 & 18.0 & 22.5 & $5.0-9.0$ \\
\hline
\end{tabular}

a: IOM pre-pregnancy BMI category: underweight (BMI < 18.5), normal weight (BMI 18.5-24.9), overweight (BMI 25-29.9), obese (BMI $\geq 30$ );

b: Asian pre-pregnancy BMI category: underweight (BMI < 18.5), normal weight (BMI 18.5-22.9), overweight (BMI 23-24.9), obese (BMI $\geq 25)$

Note: Shaded areas represent the 2009 IOM gestational weight gain ranges

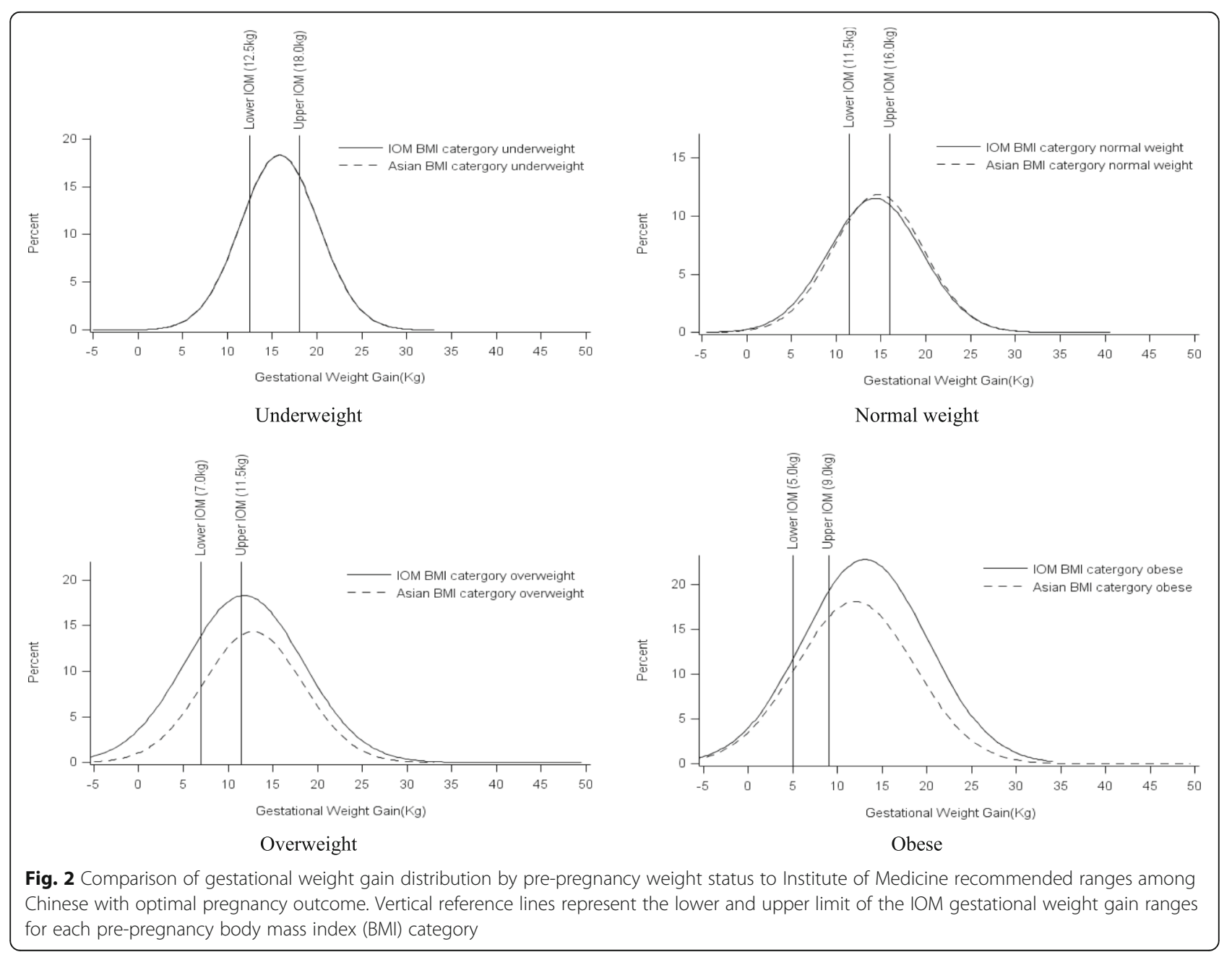


Table 4 Comparison of adverse pregnancy outcomes between subjects with optimal GWG determined by the Asian BMI cut-offs ${ }^{\#}$ and those with optimal GWG determined by the IOM BMI cut-offs ${ }^{\#}$

\begin{tabular}{|c|c|c|c|c|c|c|}
\hline \multirow[t]{2}{*}{ Outcomes } & \multicolumn{2}{|c|}{$\begin{array}{l}\text { Subjects with optimal GWG according to } \\
\text { Asian BMI category }\end{array}$} & \multicolumn{2}{|c|}{$\begin{array}{l}\text { Subjects with optimal GWG according to } \\
\text { IOM BMI category }{ }^{\#}\end{array}$} & \multirow[t]{2}{*}{$\begin{array}{l}\text { Crude OR } \\
(95 \% \mathrm{Cl})\end{array}$} & \multirow[t]{2}{*}{$\begin{array}{l}\text { Adjusted OR } \\
(95 \% \mathrm{Cl})^{\mathrm{a}}\end{array}$} \\
\hline & $\mathrm{N}$ & $\%$ & $\mathrm{~N}$ & $\%$ & & \\
\hline \multicolumn{7}{|l|}{ Preterm } \\
\hline Yes & 434 & 7.10 & 450 & 6.99 & $1.02(0.89,1.17)$ & $1.03(0.90,1.19)$ \\
\hline No & 5676 & & 5988 & & Reference & \\
\hline \multicolumn{7}{|l|}{ Birth weight } \\
\hline LBW & 351 & 5.74 & 370 & 5.75 & $0.99(0.85,1.15)$ & $1.01(0.87,1.17)$ \\
\hline Macrosomia & 242 & 3.96 & 319 & 4.95 & $0.79(0.67,094)$ & $0.79(0.67,0.94)$ \\
\hline Normal & 5517 & & 5749 & & & \\
\hline \multicolumn{7}{|c|}{ Birth weight by gestational age } \\
\hline SGA & 520 & 8.51 & 530 & 8.23 & $1.02(0.90,1.16)$ & $1.02(0.90,1.16)$ \\
\hline LGA & 530 & 8.67 & 641 & 9.96 & $0.86(0.76,0.97)$ & $0.86(0.76,0.98)$ \\
\hline Normal & 5060 & & 5267 & & & \\
\hline \multicolumn{7}{|l|}{$\mathrm{PIH}$} \\
\hline Yes & 165 & 2.70 & 190 & 2.95 & $0.91(0.74,1.13)$ & $0.93(0.75,1.15)$ \\
\hline No & 5945 & & 6248 & & & \\
\hline \multicolumn{7}{|l|}{ GDM } \\
\hline Yes & 57 & 0.93 & 57 & 0.89 & $1.05(0.73,1.52)$ & $1.06(0.73,1.53)$ \\
\hline No & 6053 & & 6381 & & & \\
\hline
\end{tabular}

${ }^{a}$ Adjustment covariates are maternal age, parity, education and smoking during pregnancy

\#Optimal gestational weight gain determined by pre-pregnancy BMI category is listed in Table 1

compared the ORs for abnormal birth weight and abnormal GWG determined using the IOM-recommended ranges and abnormal GWG determined using the quartile ranges observed from the study sample. He concluded that a GWG above the IOM recommendations might not be helpful for Chinese women. Our data support his finding that the IOM recommendations are narrower than the observed distribution and that the upper limits of the distribution are too restrictive for Chinese women. However, the IOM-recommended GWG ranges are still within the middle $70 \%$ of Chinese GWG distributions in both BMI classification schemes. Elevating the upper limit of the GWG ranges may lead to long-term adverse outcomes, including postpartum weight retention and future overweight and obesity in mother or offspring. Neither the previous studies [9-13] nor our study include data on postpartum outcomes. Therefore, whether Chinese women could gain more above the upper end of the IOM guidelines needs further investigation.

Previous researchers found that the GWG distribution among specific populations would vary by different pre-pregnancy BMI classification schemes [21, 22]. Our study found that this distribution variation also exists among Chinese women. This is consistent with previous findings $[10,11]$ that, based on the Asian BMI classification, found that the mean GWG among Chinese women decreased as pre-pregnancy BMI increased. However, based on the IOM BMI classification, we found that the relationship between GWG and pre-pregnancy BMI followed a U-shaped curve, which is inconsistent with Jiang's result [12]. The sample sizes of women with a pre-pregnancy BMI greater than 30 strata in our study $(n=170)$ and Jiang's study $(n=58)$ are both small. The reason for this inconsistency needs further research.

\section{BMI classification for GWG monitoring among Chinese women}

Before 2018, no universal BMI cut-off points were recommended in China, and there were potential differences in the care of different BMI groups at the participating hospitals. The recommended weight gain ranges during pregnancy would vary when using different pre-pregnancy BMI cut-off points [5]. Our study found that women with an optimal GWG determined by the Asian BMI cut-off points had lower risks of macrosomia $(\mathrm{OR}=0.78,95 \% \mathrm{CI}: 0.67,0.94)$ and LGA $(\mathrm{OR}=$ $0.86,95 \% \mathrm{CI}: 0.76,0.98)$ than women with an optimal GWG determined by IOM BMI cut-off points suggests that the Asian BMI cut-off points are more appropriate for Chinese women. In 2004, WHO experts declared that Asian populations have different associations between BMI and health risks than Western populations do [23]. Recommending GWG ranges based on the IOM BMI cut-off points would classify some Chinese women 
in lower pre-pregnancy BMI categories and suggest them gain more weight than necessary, which may put them at an unnecessarily higher risk for macrosomia and LGA [3]. In our sample, $13.42 \%$ of the women were misclassified as normal weight, and $8.37 \%$ were misclassified as overweight if the pre-pregnancy weight status was determined by the IOM BMI cut-off points instead of the Asian cut-off points. In Jan 2018, the national GWG guideline for Chinese women was published [8], and a universal recommendation with the IOM criteria may result in more misclassification and unnecessary weight gain among pregnant Chinese women in the future.

\section{Strengths and limitations}

Strengths and limitations should be considered when interpreting the study findings. Information on birth weight and maternal complications was obtained from medical records, which minimized the potential misclassification of the outcomes. Information on gestational age and infant gender was available, which allowed us to not only control for gestational age when studying the relationship with LBW and macrosomia but also examine the association with SGA and LGA. Furthermore, the data contained detailed information on maternal demographic information, which allowed adjusting for several important potential confounding factors simultaneously. However, the limitation of our study should be considered when interpreting the study findings. Although weight at delivery was measured at the hospital and extracted from discharge abstracts, pre-pregnancy weight was recorded based on self-reported information. There is a potential misclassification for pre-pregnancy BMI status. However, high correlations were found between self-report and measured pre-pregnancy weight [24]. Our data are not from a nationally representative sample, we only used a retrospective cohort study in Changsha, which is in the central region of China, to eliminate the potential for selection bias. The generalizability of our findings and the appropriateness of the GWG guidelines by the Chinese Society of Gynecology and Obstetrics need further investigation.

\section{Conclusion}

The IOM-recommended GWG ranges are within the middle $70 \%$ of the distributions in Chinese women. Pre-pregnancy weight status should be determined using the Asian BMI cut-off points when applying IOM GWG recommendations to Chinese women.

\section{Additional files}

Additional files 1: Table S1. Subgroup analysis of comparison of adverse pregnancy outcomes between different gestational weight gain groups in the pre-pregnancy BMl 23.0-24.9 strata. (DOC $47 \mathrm{~kb}$ )
Additional files 2: Table S2. Subgroup analysis of comparison of adverse pregnancy outcome between different gestational weight gain groups in the pre-pregnancy BMI 25-29.9 strata. (DOC 47 kb)

\section{Abbreviations}

BMI: Body mass index; Cl: Confidence intervals; DBP: Diastolic blood pressure; GDM: Gestational diabetes mellitus; GWG: Gestational weight gain; IOM: Institute of Medicine; LGA: Large-for-gestational age; OR: Odds ratio; $\mathrm{PIH}$ : Pregnancy-induced hypertension; SBP: Systolic blood pressure; SGA: Small-for-gestational age

\section{Acknowledgements}

We thank the health workers in the Maternity and Child Care Hospital in the Tianxin and Yuelu districts for their assistance in the fieldwork. The first author Xin Huang is a new investigator in Hunan Normal University.

\section{Ethical approval and consent to participate}

This study was reviewed by the Ethical and Confidentiality Committee of Central-South University (Reference 14/SPH/1005 (approved on 20th Oct 2015)), both institution review boards (IRB) from the Maternity and Child Care Hospital (MCCH) of Yuelu District (IRB of MCCH of Yuelu District) and Tianxin District (IRB of MCCH of Tianxin District). Written consent has been signed by all participants. All personal information has been recoded with initials or numbers which guaranteed no information of the participants can be identified in data analysis.

\section{Funding}

This work was funded by Natural Science Foundation of Hunan Province (2017JJ3215), Open Project of Key Laboratory of Environmental Pollution Monitoring and Disease Control, Ministry of Education, Guizhou Medical University (GMU-2017-005) and Zhishan Plan Program of the Third Xiangya Hospital, Central South University ([2017]15). The funding had no role in study design, data collection, data analysis, data interpretation, or writing the report.

Availability of data and materials

The datasets used and analyzed during the current study are available from the corresponding author on reasonable request.

\section{Authors' contributions}

$\mathrm{XH}, \mathrm{HT}$, and $\mathrm{J}$ designed the research; $\mathrm{XH}, \mathrm{TS}, \mathrm{MC}$ and $\mathrm{CM}$ conducted the cohort study; $\mathrm{XH}$ performed statistical analysis; $\mathrm{XH}, \mathrm{HT}, \mathrm{TS}, \mathrm{MC}, \mathrm{CM}$ and $\mathrm{L}$ wrote the first draft and all authors contributed to the final draft and approved the manuscript.

Competing interests

The authors declare that they have no competing interests.

\section{Publisher's Note}

Springer Nature remains neutral with regard to jurisdictional claims in published maps and institutional affiliations.

\section{Author details}

${ }^{1}$ Department of Preventive Medicine, School of Medicine, Hunan Normal University, Changsha, Hunan, China. ${ }^{2}$ Department of Epidemiology and Health Statistics, Xiangya School of Public Health, Central South University, Changsha, Hunan, China. ${ }^{3}$ Maternity and Child Care Hospital of Yuelu District, Changsha, Hunan, China. ${ }^{4}$ Maternity and Child Care Hospital of Tianxin District, Changsha, Hunan, China. ${ }^{5}$ Department of Obstetrics and Gynecology, The Third Xiangya Hospital of Central South University, 138 Tongzipo Road, Yuelu District, Changsha 410013, Hunan, Province, China.

Received: 21 January 2018 Accepted: 17 May 2018

Published online: 29 May 2018

References

1. Adair LS. Long-term consequences of nutrition and growth in early childhood and possible preventive interventions. Nestlé Nutr Inst Workshop Ser. 2014;78:111-20. 
2. Shao Y, Qiu J, Huang H, Mao B, Dai W, He X, et al. Pre-pregnancy BMI, gestational weight gain and risk of preeclampsia: a birth cohort study in Lanzhou, China. BMC Pregnancy Childbirth. 2017;17(1):400.

3. Baugh N, Harris DE, Aboueissa AM, Sarton C, Lichter E. The impact of maternal obesity and excessive gestational weight gain on maternal and infant outcomes in Maine: analysis of pregnancy risk assessment monitoring system results from 2000 to 2010. J Pregnancy. 2016;2016:5871313.

4. Davis RR, Hofferth SL. The association between inadequate gestational weight gain and infant mortality among U.S. infants born in 2002. Matern Child Health J. 2012;16(1):119-24.

5. Institute of Medicine (US) and National Research Council (US) Committee to Reexamine IOM Pregnancy Weight Guidelines, Rasmussen KM, Yaktine AL. Weight gain during pregnancy: reexamining the Guidelines. Washington, DC: National Academies Press (US); 2009.

6. Parker JD, Abrams B. Prenatal weight gain advice: an examination of the recent prenatal weight gain recommendations of the Institute of Medicine. Obstet Gynecol. 1992;79:664e669.

7. Edwards LE, Hellerstedt WL, Alton IR, Story M, Himes JH. Pregnancy complications and birth outcomes in obese and normal-weight women: effects of gestational weight change. Obstet Gynecol. 1996;87:389e394.

8. Obstetrics Subgroup, Chinese Society of Obstetrics and Gynecology, Chinese Medical Association. Guideline of preconception and prenatal care (2018) (Article in Chinese). Zhonghua Fu Chan Ke Za Zhi. 2018;53(1):7-13.

9. Yang YD, Yang HX. Investigation into the clinical suitability of Institute of Medicine 2009 guidelines regarding weight gain during pregnancy for women with full term singleton fetus in China. Zhonghua Fu Chan Ke Za Zhi. 2012;47(9):646-50.

10. Zhou YB, Li HT, Ye RW, Li ZW, Zhang YL, Zhang L, et al. Gestational weight gain, cesarean delivery, and cesarean delivery on maternal request: a cohort analysis of Chinese nulliparous women. Ann Epidemiol. 2017;27(2):96-102.e3.

11. Wong W, Tang NL, Lau TK, Wong TW. A new recommendation for maternal weight gain in Chinese women. J Am Diet Assoc. 2000;100(7):791-6.

12. Jiang $X$, Liu M, Song Y, Mao J, Zhou M, Ma Z, et al. The Institute of Medicine recommendation for gestational weight gain is probably not optimal among non-American pregnant women: a retrospective study from China. J Matern Fetal Neonatal Med. 2017;27:1-6.

13. Yang S, Peng A, Wei S, Wu J, Zhao J, Zhang Y, et al. Pre-pregnancy body mass index, gestational weight gain, and birth weight: a cohort study in China. PLoS One. 2015;10(6):e0130101.

14. Ko GTC, Chan JC, Cockram CS, Woo J. Prediction of hypertension, diabetes, dyslipidaemia or albuminuria using simple anthropometric indexes in Hong Kong Chinese. Int J Obes. 1999;23:1136-42.

15. Deurenberg-Yap M, Chew SK, Lin FP, van Staveren WA, Deurenberg P. Relationships between indices of obesity and its comorbidities among Chinese, Malays and Indians in Singapore. Int J Obes. 2001;25:1554-62.

16. Dai L, Deng C, Li Y, Zhu J, Mu Y, Deng Y, et al. Birth weight reference percentiles for Chinese. PLoS One. 2014;9(8):e104779.

17. Duryea EL, Hawkins JS, McIntire DD, Casey BM, Leveno KJ. A revised birth weight reference for the United States. Obstet Gynecol. 2014;124(1):16-22.

18. Khanolkar AR, Hanley GE, Koupil I, Janssen PA. 2009 IOM guidelines for gestational weight gain: how well do they predict outcomes across ethnic groups? Ethn Health. 2017;13:1-16.

19. Choi SK, Lee G, Kim YH, Park IY, Ko HS, Shin JC. Determining optimal gestational weight gain in the Korean population: a retrospective cohort study. Reprod Biol Endocrinol. 2017;15(1):67.

20. Nomura K, Kido M, Tanabe A, Nagashima K, Takenoshita S, Ando K. Investigation of optimal weight gain during pregnancy for Japanese women. Sci Rep. 2017;7(1):2569.

21. Olson CM, Strawderman MS, Hinton PS, Pearson TA. Gestational weight gain and postpartum behaviors associated with weight change from early pregnancy to 1 y postpartum. Int J Obes Relat Metab Disord. 2003:27:117e127.

22. Fernandez ID, Hoffmire CA, Olson CM. Gestational weight gain in adolescents: a comparison to the new Institute of Medicine recommendations. J Pediatr Adolesc Gynecol. 2011;24(6):368-75.

23. WHO Expert Consultation. Appropriate body-mass index for Asian populations and its implications for policy and intervention strategies. Lancet. 2004;363(9403):157-63.

24. Headen I, Cohen AK, Mujahid M, Abrams B. The accuracy of self-reported pregnancy-related weight: a systematic review. Obes Rev. 2017;18(3):350-69.

\section{Ready to submit your research? Choose BMC and benefit from:}

- fast, convenient online submission

- thorough peer review by experienced researchers in your field

- rapid publication on acceptance

- support for research data, including large and complex data types

- gold Open Access which fosters wider collaboration and increased citations

- maximum visibility for your research: over $100 \mathrm{M}$ website views per year

At BMC, research is always in progress.

Learn more biomedcentral.com/submissions 